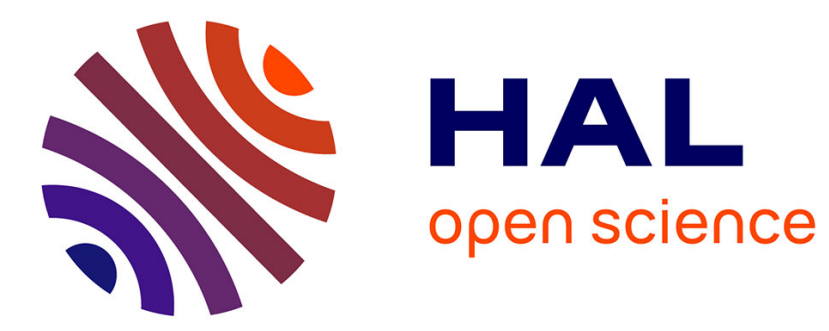

\title{
Enjeux du travail pédagogique en petit groupe pour des enfants ou des adolescents malades en milieu hospitalier
}

Danièle Toubert-duffort

\section{To cite this version:}

Danièle Toubert-duffort. Enjeux du travail pédagogique en petit groupe pour des enfants ou des adolescents malades en milieu hospitalier. La nouvelle revue de l'adaptation et de la scolarisation, 2017, 79-80, pp.89-105. 10.3917/nras.079.0089 . hal-01673856

\section{HAL Id: hal-01673856 \\ https://hal-inshea.archives-ouvertes.fr/hal-01673856}

Submitted on 22 Dec 2021

HAL is a multi-disciplinary open access archive for the deposit and dissemination of scientific research documents, whether they are published or not. The documents may come from teaching and research institutions in France or abroad, or from public or private research centers.
L'archive ouverte pluridisciplinaire HAL, est destinée au dépôt et à la diffusion de documents scientifiques de niveau recherche, publiés ou non, émanant des établissements d'enseignement et de recherche français ou étrangers, des laboratoires publics ou privés. 


\title{
Enjeux du travail pédagogique en petit groupe pour des enfants ou des adolescents malades en milieu hospitalier
}

\author{
Danièle TOUBERT-DUFFORT \\ Maître de conférences en psychologie clinique \\ INS HEA - Grhapes (EA 7287)
}

\begin{abstract}
Résumé: La visée de cet article est d'analyser les enjeux d'un travail de mise en groupe dans un contexte d'enseignement en milieu hospitalier, pour des élèves gravement malades et hospitalisés. La littérature montre que l'impact d'une maladie grave sur le développement psycho-social de l'enfant prend plusieurs formes qui, en se conjuguant, peuvent conduire à un retard dans le développement social et émotionnel. Partant de l'hypothèse que le travail en petit groupe offre aux jeunes malades la possibilité d'apprendre par l'expérience socio-émotionnelle et relationnelle, nous inscrivons notre recherche dans une perspective à la fois clinique et socio-constructiviste. Notre méthodologie a consisté à analyser le contenu des entretiens menés avec les jeunes et avec l'enseignant, ainsi que les observations des séances collectives, filmées. Les adolescents concernés sont âgés de 14 à 16 ans et scolarisés en milieu hospitalier. II s'agit de s'intéresser à la façon dont les adolescents vivent et donnent sens à leur expérience subjective, à ce que mobilise en chacun l'entrée dans un nouveau groupe, et aux liens qui se tissent dans cette constellation groupale (expérience collective). Nous montrons que les postures de l'enseignant, qui s'ajustent dans l'activité à ce qu'il perçoit du côté du sujet, du côté des interactions et du côté du groupe, nous renseignent autant sur ses intentions pédagogiques que sur la dimension imaginaire, également constitutive, du lien.
\end{abstract}

Mots-clés: Adolescent - Apprentissages - Élève malade - Expérience subjective - Groupe pédagogique Postures de l'enseignant - Processus groupal.

\section{Challenges of small group teaching for sick children or teenagers in hospital}

Summary: The aim of this article is to analyze the process of group constitution in the context of education in a hospital environment, for seriously sick and hospitalized pupils. The literature shows that the impact of a serious illness on the psychosocial development of the child takes several forms which, by conjugating, can lead to a delay in the social and emotional development. Assuming that the work in small groups offer to the young sick the possibility of learning by the socio-emotional and relational experience, we register our search in a prospect at the same time clinical and socio-constructivist. Our methodology consisted in analyzing the contents of the interviews led with the young people and with the teacher, as well as the observations of the collective, filmed sessions. Our methodology consisted in analyzing the contents of the conversations (maintenances) led with the young people and with the teacher, as well as the observations of the collective, filmed sessions. The concerned teenagers are from 14 to 16 years old and schooled in a hospital environment. We were interested in the way in which the adolescents interpret and give sense to their subjective experience, to what entering a new group mobilizes in them, as well as to the ties which are established within the group (collective experience). The results showed that the teacher's postures that he adjusted according to what he perceived of each adolescent, their interactions, and the group are revealing of both his pedagogical intentions and the imaginary dimension, which also contributes to the constitution of ties.

Keywords: Adolescent - Education group - Group process - Learning process - Sick pupils - Subjective experience - Teachers's posture. 
ET article aborde les enjeux d'une mise en groupe d'élèves malades, scolarisés au sein d'une Unité d'enseignement (UE) dans un établissement spécialisé ou à l'hôpital. II s'agit d'analyser les raisons et les conditions pédagogiques et cliniques qui permettent à ces élèves de tirer profit des situations collectives proposées par leur enseignant afin d'apprendre en groupe mais également apprendre de l'expérience collective, c'est-à-dire apprendre du groupe.

\section{CONTEXTUALISATION DE LA PROBLÉMATIQUE}

\section{Scolarisation des élèves malades et pratiques pédagogiques: entre maintien du lien social et maintien des liens de pensée}

Au sein des établissements des secteurs médico-social et sanitaire, l'accompagnement des enfants et des adolescents souffrant de pathologies sévères à la suite d'un accident (traumatisme crânien) ou d'une maladie grave (accident vasculaire cérébral, tumeurs cérébrales, cancers...) confronte les professionnels de l'éducation à la nécessité d'un double aménagement: celui de la scolarisation de ces jeunes, et celui des pratiques pédagogiques.

D'une part, la scolarisation est aménagée de façon à concilier les impératifs qui s'imposent à l'enfant ou à l'adolescent (rééducations, soins, appareillages parfois, douleur, fatigabilité, déficiences, restriction de l'autonomie...). Le recours nécessaire aux traitements, I'organisation des prises en charge et la logique médicale impliquent le risque iatrogène de voir disparaître l'élève derrière le patient. La préservation d'un espace social dans lequel le jeune puisse, pour lui-même et en lui-même, penser, imaginer, apprendre, et dans le même temps éprouver l'impact de son action et de sa parole au regard d'autrui, constitue dans ce contexte un élément fondamental. Lorsque la scolarisation se déroule depuis plusieurs années au sein d'un établissement médicalisé dans lequel les jeunes résident également, les enjeux sont de taille: faire de l'espace scolaire un espace social suffisamment ouvert sur l'extérieur et assumer, sur le plan de la pensée et de la pratique, en particulier dans le positionnement à l'égard de l'enfant ou de l'adolescent, l'écart entre prendre en charge et accompagner un élève en situation de maladie et/ou de handicap.

D'autre part, l'enseignant est amené à repenser ses pratiques, dans un double mouvement d'implication (émotionnelle, cognitive) et de distanciation. Son positionnement au sein d'une équipe pluri-professionnelle mais parfois aussi exclusivement médicale (quand il exerce seul en contexte hospitalier) le confronte à d'autres points de vue, d'autres référents, d'autres logiques, l'amenant à se décaler régulièrement, au risque parfois d'en oublier sa tâche de base (enseigner, éduquer). L'enjeu professionnel est alors de maintenir les conditions d'une mise en mouvement de la pensée, afin de se dégager des positions mortifères susceptibles d'être actualisées dans le lien soignant-patient ou dans le lien maître-élève, par exemple du contrôle excessif ou d'une emprise immodérée à l'égard de jeunes particulièrement vulnérables (Toubert-Duffort, 2009).

Dans le contexte d'une structure médicalisée qui développe une logique de prise en charge, essentiellement individuelle et rééducative, la pratique de l'enseignement est ainsi guidée par un double objectif : réintroduire la dimension sociale dans l'acte 
d'apprendre, et créer les conditions favorables pour permettre à l'enfant malade d'éprouver le plaisir d'une pensée autonome, malgré les contraintes liées à son état de santé et en dépit du contexte traumatogène.

\section{Groupes et apprentissages, quels enjeux pour l'élève malade scolarisé en milieu hospitalier?}

Deux grandes perspectives psychosociologiques et développementales soutiennent la thèse d'une origine sociale des processus mentaux et psychiques.

L'une, socio-constructiviste, inspirée des travaux de Lev. Vygotski (1934) et prolongée par ses disciples (Bruner, 1991; Clot, 2010 ; Doise et Mugny, 1981; Perret-Clermont et Nicolet, 2001) considère que "le développement individuel des fonctions mentales supérieures relève d'un processus de genèse sociale se produisant au cours de pratiques sociales "(Roux, 2003). Dans cette perspective, le développement cognitif est un produit de l'apprentissage, et l'ensemble des fonctions psychiques supérieures sont issues de la transformation de processus sociaux (interpersonnels) en processus cognitifs (intra-personnels). Les variables sociales sont indissociables des processus constructeurs eux-mêmes. Le travail de groupe constitue, pour ces auteurs, un environnement sociocognitif susceptible, à certaines conditions, de générer des progrès individuels.

L'autre perspective, clinique, inspirée des travaux de Sigmund Freud (1921), considère que le sujet prend naissance dans le groupe (groupe primaire), s'individualise de manière plus ou moins complète, et construit sa singularité de cette base partagée. Cette base culturelle du " Je » détermine les limites de l'individu et du groupe, du moi et du non-moi, de l'intérieur et de l'extérieur, du réel et de l'imaginaire. Dans les groupes d'appartenance " secondaires " (comme la classe), c'est la différence qui organise les échanges, l'intrapsychique et l'intersubjectif s'articulant en un double mouvement. Cette expérience permet ainsi de compléter l'intériorisation culturelle et de concrétiser l'appréhension du temps et de l'espace, les groupes secondaires remplissant une fonction de socialisation et d'intériorisation des normes et des valeurs (Rouchy, 2009): le " Je » existe au regard d'autrui et dans le rapport de soi à soi, situé à une place, dans un réseau groupal. C'est dans cette approche, qui souligne la nature à la fois individuelle et collective de l'identité, que nous nous situons. Ce positionnement épistémologique nous conduit à considérer le groupe pédagogique comme un espace dynamique, à la fois potentiellement support d'identifications, de projections imaginaires, mais aussi de différenciations. II nous amène à nous interroger sur la construction même de l'espace groupal, la place que chacun y occupe, y compris l'enseignant, ou encore sur celle attribuée au plan fantasmatique. La littérature montre que l'impact d'une maladie grave sur le développement psycho-social de l'enfant prend plusieurs formes qui, en se conjuguant, peuvent conduire à un retard dans le développement social et émotionnel. Faisant irruption dans le déroulement d'une vie, la maladie grave de l'enfant ou de l'adolescent, en confrontant le sujet à un contexte inédit, bouleverse en profondeur ses repères internes et externes, et provoque des modifications importantes de sa subjectivité, dans le sens de la perte mais aussi de la créativité (Toubert, 2016). Les centres d'intérêt se modifient, la curiosité semble parfois se restreindre ou au contraire se développer autour de nouveaux objets. 
Après l'état de sidération (gel de la pensée) lié au traumatisme primaire, l'enjeu principal pour le jeune malade, enfant ou adolescent, se situe dans ses possibilités de "remise en mouvement " des processus secondaires (Doudard, Riquin, Malka, Avarello et Duverger, 2014), témoignage du travail de la maladie (Pédinielli, 1987, 1990) ou travail de la souffrance (Gutton, 1979) qui s'opère en lui. Ces concepts ont été forgés pour désigner les phénomènes psychologiques qui accompagnent la maladie somatique: la maladie ne supposerait pas une immobilisation des processus psychiques mais susciterait au contraire une économie spécifique et une modification de la subjectivité, tant dans le rapport au corps et à la maladie que dans la relation à I'histoire personnelle. La manière dont le sujet parle de sa maladie, la vit, en gère les aléas, lui donne un sens, se comporte en réaction à celle-ci, traduisent la dimension active du rapport à la maladie. Comportant un caractère de nécessité, et composé d'une succession de phases entraînant la production de représentations après transformation - sous douleur psychique - de l'état antérieur, le travail de la maladie comporte des effets d'élaboration et, pour reprendre le modèle freudien du travail de deuil, des effets de libération.

Ayant à subir un parcours thérapeutique lourd, le jeune malade connait ainsi une réalité de vie qui l'éloigne des enfants de son âge, et subit de fait une situation d'isolement, voire de stigmatisation. Sur le plan psychique, le sentiment d'être différent de ses pairs, exacerbé par les marques corporelles générées par la maladie (perte de cheveux, prise de poids, amputation, cicatrice...) s'accompagne bien souvent d'une crainte de perdre sa place dans les groupes d'affiliation, groupe de copains, groupe familial... Le handicap invisible, en particulier les séquelles neurocognitives ou psychologiques des pathologies touchant la sphère cérébrale, n'est pas plus facile à vivre sur le plan de l'imaginaire social: il perturbe le rapport au monde et à soi, et provoque chez le jeune cérébro-lésé un vécu d'étrangeté et de non-conformité (Toubert-Duffort, 2011). Le langage est fortement perturbé, à la fois sur le plan instrumental à cause des lésions, et sur le plan communicationnel dans la mesure où l'expérience, du fait de sa proximité avec la mort, est difficile à mettre en mots, voire incommunicable (Oppenheim, 2003).

Ainsi ces enfants ou ces adolescents peuvent-ils montrer des particularités de fonctionnement, liées à leur vécu traumatique, au travail psychique qui s'opère en eux, aux séquelles neuromotrices, neurocognitives ou psychologiques de leur pathologie, aux effets iatrogènes de l'hospitalisation et des traitements, et enfin au contexte lui-même. Cette situation est d'autant plus difficile à vivre à l'âge de l'enfance et de l'adolescence qui sont des périodes de la vie où l'on accorde une grande importance à l'apparence physique et à l'acceptation sociale. Nous voyons combien l'élève malade a besoin d'un espace médiatisé lui offrant la possibilité d'échanges et d'expériences relationnelles diversifiées avec les pairs de génération, de manière à l'aider à construire du sens sur son expérience et à se l'approprier. II a également besoin d'être accompagné pour relativiser la place de la maladie dans sa vie, et retrouver confiance dans ses capacités. Que peut apporter le groupe pédagogique, dans un tel contexte?

Le groupe pédagogique constitue potentiellement un espace d'étayage et de médiation grâce à la complémentarité des conduites et aux possibilités d'identification offertes par chacun à chacun. Le contexte collectif rend également possible, du fait 
de l'hétérogénéité des membres, les confrontations de point de vue et la mise en question de ses propres positions. À la faveur des jeux identificatoires, les situations peuvent ainsi être envisagées de manière renouvelée. Dans le contexte de la maladie, le groupe pédagogique peut " permettre à chacun de se reconnaitre dans les autres et de mieux contenir et transformer le sentiment d'être devenu un étranger au soi antérieur, coupé de la communauté humaine normale " (Toubert-Duffort et Sirota, 2016). Par ailleurs le groupe pédagogique, s'il offre des conditions de sécurité suffisante par l'instauration d'un cadre stable et contenant, constitue un espace d'apprentissage par l'expérience dialectique de la distance relationnelle, où le rôle d'enseignant et le rôle d'élève sont fonctionnels et complémentaires (Pichon-Rivière, 1971). Ainsi le dispositif de groupe pédagogique permet-il, à certaines conditions, de travailler au moins deux dimensions de l'apprentissage:

- la tâche informative qui concerne la connaissance de certains contenus (culturel, d'ordre instrumental, scolaire, etc.): apprendre en groupe;

- la tâche formative, en partie implicite, qui concerne la sensibilisation au fonctionnement groupal et, corrélativement pour l'enfant ou l'adolescent, l'intérêt pour son mode de relation aux autres, l'ouverture à sa propre vie intérieure, et la découverte de sa manière particulière d'aborder les objets et le lien entre les objets: apprendre du groupe.

Si les conditions le permettent, le travail en groupe mené dans le contexte de l'unité d'enseignement ou de l'hôpital offre ainsi aux jeunes la possibilité de se retrouver entre soi, de s'entraider, d'apprendre par l'expérience relationnelle, d'éprouver l'impact de ses positions et de sa parole, et d'exercer une forme d'autonomie.

\section{DISPOSITIF DE RECHERCHE}

\section{Objectifs, hypothèses}

En nous appuyant sur une recherche menée dans un groupe-classe au sein d'une Unité d'enseignement (UE) à l'hôpital, nous questionnons dans cet article les liens entre le travail psychique des élèves, leurs éventuelles progressions, au regard de l'expérience relationnelle qu'implique le groupe, et les postures de l'enseignant. Nous cherchons à préciser pourquoi et à quelles conditions, des adolescents souffrant de pathologies graves (traumatisme crânien sévère, AVC, tumeurs cérébrales, cancers...), scolarisés dans une unité d'enseignement en milieu hospitalier, tirent profit des situations collectives proposées par leur enseignant afin, non seulement d'apprendre à plusieurs, mais également d'apprendre de l'expérience collective. Nous posons ainsi l'hypothèse que ces situations collectives, y compris pour des élèves atteints dans leurs processus de pensée et de socialisation, peuvent, à certaines conditions, soutenir des processus de reconstruction (réaménagements identitaires positifs, en particulier développement de l'estime de soi, exercice de l'autonomie de pensée, sentiment de continuité), favoriser les liens de pensée, le plaisir d'apprendre, et enfin la socialisation (Toubert-Duffort, 2012).

\section{Population, contexte et méthodologie de la recherche}

La recherche, menée sur douze mois, a été conduite au sein d'un groupe-classe composé de 5 à 7 adolescents selon les périodes (2 garçons, 5 filles) âgé(e)s de 
14 à 16 ans: à l'exception de Joseph, 14 ans, qui vit avec une maladie à pronostic vital engagé (récidive de cancer) et de Lucie, 14 ans, consciente d'une possible récidive après un accident vasculaire cérébral, l'état de santé des autres jeunes est stable, mais nécessite de lourdes prises en charge rééducatives, médicamenteuses, et parfois un appareillage de pointe. Certains de ces élèves sont en situation de handicap moteur, entravés dans leur autonomie de déplacement et d'expression à cause des séquelles neuromotrices et neurocognitives d'un traumatisme crânien pour trois d'entre eux (Marine, 15 ans; Julien, 16 ans et Chloé, 15 ans), et d'une tumeur cérébrale d'origine cancéreuse pour deux autres (Laura, 14 ans, Amélie, 15 ans). L'équipe pédagogique de l'unité hospitalière a considéré que le travail de cette recherche relevait de la " tâche primaire " (Kaës, 2003) de l'enseignant, chercheurs-praticiens et praticiens-chercheurs étant conjointement impliqués dans une démarche qui visait non seulement à produire un savoir sur des pratiques, mais également à accompagner le déploiement et/ou l'évolution de ces pratiques. Nous avons questionné les enjeux mobilisés au sein du groupe pédagogique, animé par un enseignant spécialisé, le groupe étant impliqué sur les deux niveaux d'apprentissage précédemment énoncés, celui de la tâche informative, et celui de la tâche formative (Pichon, 1971).

La méthodologie s'appuie sur deux axes principaux: le premier axe porte sur le vécu des élèves, s'agissant du groupe-classe, de leur rapport à autrui, de leur place dans le groupe. Comment ces sept élèves ont-ils vécu leur entrée et leur travail en groupe, quel sens donnent-ils à cette expérience collective en classe, qu'ont-ils à en dire, de ce qu'elle leur apporte ou non? Que leur font éprouver les différentes situations collectives expérimentées en classe? Comment perçoivent-ils le positionnement de l'enseignant? Le leur dans le groupe?

Le second axe s'intéresse à l'analyse des processus mobilisés par les situations pédagogiques de groupe, et à l'apport qu'elles constituent pour chacun, dans le contexte particulier de l'hospitalisation et de la maladie. II s'agit de préciser, dans une perspective diachronique, quels sont les processus psychiques mobilisés lors du (dé)groupement (Kaës, 2002). Par quels procédés le groupe s'organise ou se désorganise-t-il ? Comment le sujet participe-t-il de cette (dé)liaison?

Nous interrogerons le positionnement de l'enseignant, garant du groupe, et plus précisément ses postures. Comment l'enseignant participe-t-il (ou non) à la constitution de l'enveloppe groupale et à la dynamique qui s'instaure? Quel est le travail psychique de l'enseignant: comment contient-il ou non, par la pensée, ce qui émerge d'idées? Comment construit-il, ou non, du sens sur ce qu'il perçoit? Quelle attention et quelles tensions sont mobilisées, du côté du professionnel, dans cette délicate entreprise?

La recherche s'appuie sur le recueil et l'analyse de différents types de matériaux: des entretiens individuels avec les jeunes et avec l'enseignant mobilisé dans la recherche-action; I'observation des séances collectives; des entretiens collectifs menés à l'issue des séances de travail, pour recueillir le point de vue des élèves sur ce qu'ils ont vécu collectivement. De même, les séances font l'objet d'une analyse avec l'enseignant, dans une perspective synchronique. 


\section{RÉSULTATS ET DISCUSSION}

\section{Comment " faire groupe ", dans un contexte " ouvert "?}

La simple réunion d'individus dans un espace commun pour accomplir une tâche d'apprentissage ne se constitue pas immédiatement ni spontanément en un véritable groupe qui soit capable d'apprendre ensemble et de s'aider dans cette démarche. Différents éléments contribuent à ce que le groupe se constitue. En premier lieu, il y a les constantes de personnes, de temps, et d'espace, ainsi que la rythmicité et les règles de fonctionnement. Nous les qualifions de constantes car elles constituent des repères fixes, invariables, qui délimitent le cadre à l'intérieur duquel se développe et prend sens l'histoire de ce groupe. Des rituels et des affichages vont rendre plus tangibles, dans certains cas, ces repères.

Il y a ensuite les supports matériels de la tâche, véritables médiateurs relationnels. En ce qui concerne leur choix, l'on notera qu'il faut tenir compte, aussi bien des possibilités affectives, cognitives et évolutives des élèves, de leur état, en particulier de leur fatigabilité, de leur niveau d'éveil ou de douleur, que du degré d'anxiété qu'ils éveillent. Il semble important de choisir du matériel qui suscite la curiosité, mais en veillant à ce que la mobilisation des conduites défensives ne devienne pas un obstacle épistémologique. Le moment d'introduction de ce matériel est aussi important. II y a:

- Ceux qui sont d'emblée présents, comme propriétés du groupe.

- Ceux qui sont introduits par le professionnel pour aider à l'aménagement d'une situation groupale d'apprentissage. Dans le contexte où priment les soins, les capacités d'ajustement de l'enseignant sont requises en vue de choisir des supports d'apprentissage suffisamment mobilisateurs et souples pour impliquer l'enfant malade.

- Ceux enfin qui sont proposés par les adolescents eux-mêmes, et qui peuvent être acceptés, après discussion avec le groupe, en tenant compte de la signification particulière qu'ils revêtent dans ce moment précis de l'évolution du groupe pédagogique et de la relation entre les membres.

- II y a enfin tout au long du cheminement du groupe, les interventions des élèves entre eux et les interventions de l'enseignant qui anime et régule le groupe.

En milieu hospitalier, les constantes de temps et de personnes sont très souvent mises à mal. Le temps d'hospitalisation ne coïncide pas avec le temps scolaire (de septembre à fin juin) : il peut être bien plus réduit ou au contraire allongé, imprévisible, et toujours dépendant de l'état de santé de l'élève. La journée de classe est elle-même bien souvent fragmentée par les temps de soin ou de rééducation, les sorties et les entrées des élèves sont ainsi fréquentes, de même que leurs absences. Les élèves ne font pas tous en même temps leur entrée dans le groupe-classe, et le temps passé ensemble varie d'une personne à l'autre. Par ailleurs, pour certains, compte tenu de leurs besoins de soin et de rééducation, le temps de scolarisation se fait d'abord en individuel, au chevet, ou dans une salle spécifique. L'entrée en groupe, dans ce contexte particulier, nécessite toujours un accueil individualisé de la part de l'enseignant, et s'accompagne inévitablement, pour l'élève qui arrive comme pour ceux qui accueillent, de sentiments de doute voire d'insécurité que le professionnel 
devra contenir et transformer en sens. Ainsi repère-t-on que l'enseignant accorde, à chaque début de séance, de l'attention à l'accueil de chacun, à la présence mais aussi à l'absence des jeunes. D'où l'importance des liens avec le personnel soignant qui va informer en amont l'enseignant, des entrées, sorties et absences des jeunes patients. Le groupe-classe présente donc, à l'hôpital, la particularité d'être un groupe semi-fermé ou ouvert.

Nous allons maintenant nous intéresser aux modes d'entrée en relation, et aux vécus actualisés en chacun des membres, lors de l'entrée en groupe.

\section{L'entrée en groupe et les besoins de sécurisation: constitution d'un objet groupe pour édifier le sentiment d'appartenance et d'identité sociale}

Chaque sujet a un mode d'entrée en relation avec autrui qui lui est propre (Sirota, 2007). L'entrée en groupe constitue donc une expérience éminemment singulière.

\section{L'exemple de Marine, 15 ans}

Dans les premières séances de travail collectif, Marine, 15 ans, montre une façon bien à elle de s'installer, près de la porte, toujours le plus en retrait possible par rapport aux autres. Son regard noir et perçant suit celui de l'enseignant.

Ses interventions, peu nombreuses et centrées sur des préoccupations personnelles (I'heure de sa rééducation, son cahier d'évaluation des acquis antérieurs...) provoquent une rupture dans le travail d'élaboration qui s'opère en groupe. Les autres jeunes la tiennent à l'écart et réagissent négativement à ces interventions. Comme si Marine ne pouvait ou ne trouvait pas à s'accorder à la rythmicité du groupe, comme si elle n'arrivait pas à partager la pensée commune et à s'installer dans ce nouvel espace. L'entretien mené avec elle, quelques mois après ces observations, nous éclaire sur son propre vécu. "Quand je suis arrivée dans ce groupe, il y a un mois, j'étais tout le temps en colère. Après les autres [...] après le maître, après le travail. En colère contre tout et tout le monde. Pour dire, je ne pouvais parler à personne, je m'entendais avec personne... Je ne venais pas en classe certains jours, je voulais voir personne, j'avais peur qu'on me rejette. Et je pensais que je suis nulle. "

Paradoxalement, dans la confrontation à ce nouveau groupe, pourtant accueillant et bienveillant, elle provoque ce qu'elle redoute le plus: se voir condamnée à l'épreuve de solitude, être exclue du groupe. L'expérience de confiance dans son propre appareil à penser est, pour Kaës (2005) un « passage obligé dans toute expérience de pensée à plusieurs ». Penser dans un groupe suppose alors de pouvoir tolérer que son propre appareil à penser les pensées se prête à l'expérience potentiellement transformatrice du groupe, c'est-à-dire de pouvoir tout d'abord se penser comme sujet de groupe.

Cette confiance est en même temps la condition de la fiabilité des pensées venant des autres. II va s'agir pour Marine, dans un premier temps, de pouvoir supporter la violence que suscite en elle l'espace groupal inconnu et donc potentiellement hostile. De déposer dans le groupe des objets de son monde interne, c'est-à-dire de tolérer que les autres les transforment, ou les négligent, ou les attaquent, ou encore les enrichissent de valeurs nouvelles. 


\section{Autre exemple, celui de Julien, 16 ans}

Julien a été victime d'un accident de scooter durant ses vacances d'été, à la suite d'une imprudence qu'il a commise, et après avoir consommé de l'alcool et du cannabis avec des copains : répondant à la sonnerie du téléphone portable, il a perdu le contrôle de son scooter, venant percuter un véhicule arrivant en face. Julien a subi un traumatisme crânien sévère avec deux semaines d'un coma persistant, et s'accompagnant d'un réveil lent. À sa sortie de coma, il a bénéficié d'une rééducation intensive pour recouvrer l'usage de la parole et une mobilité complète, puis de temps scolaires en individuel avant d'intégrer un groupe-classe quelques heures par semaine, et enfin tous les jours. Dans les suites de son traumatisme crânien, il a gardé des séquelles d'une hémiplégie (une faiblesse motrice au bras gauche notamment), présente une légère aphasie de type Broca, des séquelles neurocognitives (difficultés de repérage spatio-temporel, des difficultés mnésiques, des difficultés dans le raisonnement logico-mathématique). Julien est décrit comme un adolescent attachant, séducteur, mais très envahissant sur le plan relationnel: il laisse par exemple peu de place à la parole des pairs, reste le plus souvent centré sur lui-même, et intervient de manière inadaptée (" trop " et "à mauvais escient ") dans le cadre de la classe ou dans celui de la rééducation.

Après dix mois dans le petit groupe, il commence à s'éprouver plus solide, plus serein. "C'est toujours un peu difficile quand on arrive dans un nouveau groupe. On ne sait pas bien ce qu'on va trouver, si les autres vont nous accepter, ou pas. II peut y avoir des moqueries, des regards blessants. Moi, je fais partie des handicaps qui ne guérissent pas, même si c'est invisible. Dans ce groupe, je sais maintenant que j'existe avec ce que je dis, ce que je fais. J'ai moins peur cette année parce que je sais que ça se passe bien. [...] Ce que j'aime bien dans le travail ici, c'est que chacun met sa part individuelle dans ce qu'on fait ensemble. "

L'expérience collective de la rencontre avec les pairs s'est révélée, pour cet élève, source de craintes, mais constitue aujourd'hui des motifs de satisfaction. Julien poursuit son récit en évoquant spontanément d'autres expériences sociales. "Avec les autres, à l'extérieur de la classe, je ne parle jamais des différences, de mon accident. Avant, j'en parlais et les autres me regardaient bizarrement, comme si j'étais plus des leurs. "

Laura, 14 ans dit également, avec une colère à peine contenue: " J'ai déjà expérimenté que certains utilisent les faiblesses et tentent d'en profiter pour faire du mal. Moi maintenant, j'ai compris que les gens me regardent comme une pestiférée quand je parle de ma maladie (cancer), je préfère me taire. Je voudrais être dans un trou de souris. "

L'expérience collective fait toujours éprouver la confrontation à d'autres, potentiellement hostiles. Comme jadis le regard de la mère dans lequel l'enfant trouve ce qui lui est nécessaire pour prendre conscience de lui-même (Winnicott, 1971), le regard porté par autrui constitue un miroir qui peut se révéler tout autant narcissisant que stigmatisant, selon les situations. Ce que le sujet voit dans les yeux d'autrui, c'est non seulement un reflet de lui-même, déformé par la vision de l'atteinte, mais également les sentiments d'autrui à son égard, sentiments de malaise, de rejet, de culpabilité. 
La différence visible donne tellement à voir qu'elle aveugle (Korff-Sausse, 1996). Le risque pour le sujet est alors d'être continuellement ramené à la différence visible, au détriment de la vie intérieure.

Cependant l'exemple de Julien, conscient du malaise produit par certaines de ses attitudes mais, pour autant, capable de restaurer par la parole les conditions d'une rencontre intersubjective, révèle les potentialités du sujet: l'adolescent est capable d'un certain niveau de discernement et de compréhension des faits sociaux. Cette prise de conscience, que l'expérience relationnelle a favorisée chez Julien, contribue à renforcer sa confiance en lui, à éprouver les écarts entre lui et autrui, et à favoriser l'affirmation de sa néo-identité: ainsi existe-t-il à travers ce qu'il dit, ce qu'il fait: " Je me souviens la première fois que j'ai pris la parole dans le groupe pour rendre compte de mon observation. J'étais pas très à l'aise. J'ai dit que c'était mon hypothèse mais que j'étais pas sûr. C'était bizarre comme impression. Je parle de ce qui s'est passé quand j'ai pris la parole pour donner mon point de vue. Tout le monde m'a regardé et s'est tu... On m'a écouté, et je me suis senti à la fois content, excité même, et aussi inquiet. Content de parler et d'être écouté. Mais aussi inquiet que les autres ne soient pas d'accord avec moi. "

En ce début de groupe, la crainte de ne pas être apprécié et le risque de perdre sa valeur au regard d'autrui, peuvent se voir compensés par le plaisir narcissique à être écouté, le sentiment positif d'être attendu à une place et la possibilité de participer à une élaboration commune.

\section{Les témoignages de Lucie, 14 ans, et d'Amélie, 15 ans}

Ils apportent une illustration intéressante de ce qui précède: " ce que j'aime surtout, dans le travail à plusieurs, c'est quand je donne mes idées. On donne chacun, bien sûr, ses idées. Même si on n'est pas d'accord, on s'écoute, on discute. C'est pas un qui est dans son coin, tout seul, pour personne. Ce qu'on pense, on peut le partager... Et puis, on est écouté. Ça, c'est important " (Lucie, 14 ans). " J'ai vu que le maître et les élèves m'attendaient " dit encore Amélie, une autre élève, âgée de 15 ans. "Quand je suis arrivée, j'avais un dossier à mon nom, une place pour travailler. Quand je ne venais pas en classe, les autres élèves et l'enseignant le savaient. Je voyais que tout le monde s'inquiétait pour moi, que je comptais vraiment pour eux. [...] J'ai commencé à penser à ce que je manquais en ne venant pas dans le groupe... "

Comme l'illustrent les propos de Lucie et d'Amélie, le dispositif collectif pédagogique peut contribuer au développement de liens d'apprentissage, si toutefois le groupe devient bon objet-groupe pour le sujet, c'est-à-dire s'il est internalisé comme objet suffisamment fiable, présent et bienveillant.

À l'opposé du sentiment d'isolement, d'exclusion, ou de non-conformité, que les jeunes malades éprouvent ou craignent de ressentir au contact des pairs, c'est le plaisir narcissique et la gratification qui dominent ici, et débouchent sur le sentiment de reconnaissance sociale. Ainsi l'expérience groupale à travers l'affiliation, peut-elle favoriser un processus d'identification sociale.

\section{La dynamique entre postures de l'enseignant et postures de l'apprenant: un lien complexe}

Les gestes, les paroles, et les actions de l'enseignant intriqués à ses pensées et son imaginaire, ont immanquablement des retombées, des conséquences. Nous 
nommons "posture le schème préconstruit du "penser-dire-faire " que le sujet convoque en réponse à une situation ou à une tâche scolaire donnée " (Bucheton et Soulé, 2009, p. 35). Les postures de l'enseignant ne peuvent être considérées comme ajustées et étayant les liens de connaissance qu'au vu de leurs effets, c'est-à-dire grâce à une analyse après-coup.

Ainsi les postures d'étayage de l'enseignant et les postures d'apprentissage de l'apprenant sont liées de manière dynamique, et nous renseignent, comme nous allons le voir plus loin, autant sur les intentions pédagogiques de l'enseignant que sur la dimension imaginaire, également constitutive du lien à autrui.

\section{Analyse des postures d'étayage dans ses dimensions pragmatiques}

Pour illustrer la dynamique en jeu entre postures de l'enseignant et postures des apprenants, nous nous appuyons sur l'analyse d'une courte séance. Nous cherchons à cerner l'impact des différentes postures de l'enseignant sur les apprenants, sur le lien de groupe, et sur les interactions au sein du groupe.

Le contexte situationnel est le suivant: I'enseignant engage les adolescents dans une nouvelle recherche à mener en équipe, concernant les éoliennes et les énergies renouvelables. Sa visée est à la fois informative (acquérir des connaissances sur les énergies renouvelables) et formative (assumer le rôle d'observateur du groupe, de chef d'équipe, développer des savoirs-être, des savoir-faire, une pensée réflexive chez les élèves).

Sa posture est sous-tendue par une théorie de l'apprentissage selon laquelle l'engagement des élèves, sous forme d'une mobilisation dans un travail d'équipe, constitue un apprentissage par l'expérience relationnelle. Ce travail suppose, de son point de vue, le respect d'un certain nombre de règles qui s'imposent à tous (écoute et respect mutuels), que soient définis pour chacun une tâche commune, les objectifs d'un projet commun, que soient mis en œuvre le partage des responsabilités (échanges, répartition de rôles, différenciation des tâches). Qu'enfin le groupe, étayé par l'enseignant, puisse régulièrement revenir sur son expérience, en évaluer les contours, la penser en soi mais aussi au-dehors de soi.

Assis au milieu des élèves réunis autour d'une table, l'enseignant s'adresse au petit groupe, et précise ses attentes, offrant une médiation de toutes les interactions des élèves (posture de contrôle). Le groupe est attentif. Puis l'enseignant assigne aux élèves différentes responsabilités et rôles, préparant en quelque sorte le groupe à son autonomie: il propose à Chloé, 15 ans, d'assumer le rôle du "chef d'équipe ", et rappelant qu'elle est " novice ", demande à un " expert " de l'étayer (au sens brunérien du terme). C'est Amélie, 15 ans, qui se propose spontanément pour assumer cette tâche, vécue comme un gage de confiance. L'enseignant confie à Julien, 16 ans, le rôle d'observateur du groupe, en précisant la tâche à l'ensemble du groupe (posture d'enseignement): " je voudrais que vous preniez en compte que je demande à Julien de prendre le poste d'observateur. Je vous rappelle qu'observer signifie se mettre un peu en position extérieure, et analyser comment on travaille dans un groupe ". L'enseignant s'écarte alors du groupe, se positionnant à une autre extrémité de la salle (posture de lâcher-prise). Chacun se met au travail rapidement. Chloé propose, sur suggestion d'Amélie, de définir une organisation de travail 
(recherche des différents axes de travail, répartition des rôles), collecte l'approbation du collectif (murmure approbateur du groupe), puis s'enquiert des questions auquel l'exposé sur les éoliennes répondra. Les interactions sont nombreuses. Joseph, 14 ans, propose spontanément de prendre des notes sur ce qui est décidé « pour ne pas perdre ce qui est dit ". L'ensemble du groupe est attentif, à l'écoute. Nul ne regarde l'enseignant qui reste à distance, silencieux. Chloé s'exprime difficilement du fait de ses atteintes neuro-motrices, mais chacun l'écoute avec attention. Personne ne I'interrompt. Joseph prend des notes et il reformule au groupe ce que Chloé vient de dire, s'assurant de la bonne compréhension d'Amélie et de Laura, qui fronce les sourcils. Aucun élève ne manifeste de posture de refus ou de retrait. L'hétérogénéité du groupe ne semble pas un obstacle, elle reste positive et dynamique.

Il est intéressant d'observer que la dynamique interlocutoire à l'œuvre permet à la fois de produire des mécanismes inter et intra-individuels eu égard à des phénomènes sociaux (par exemple la place occupée par les différents partenaires) et cognitifs (accroissement des connaissances et élaboration d'outils de pensée à la portée de tous) (Roux, 2003).

Les postures de l'enseignant sont diversifiées: posture de contrôle au début pour cadrer l'ensemble des tâches; posture de lâcher-prise pour respecter l'espace d'autonomie des adolescents; posture d'enseignement pour structurer les savoirs et proposer ponctuellement un apport conceptuel. L'enseignant module probablement les formes de sa médiation en fonction de ce qu'il perçoit et observe de l'activité des élèves. Le groupe avance à la fois sur le plan cognitif (planification des tâches, début de catégorisation des questions, verbalisation, explicitation de certain terme) et sur le plan social (écouter les autres, prendre la parole, reformuler, se mettre à la place d'autrui). Cependant, lors de ce début de séance, le groupe n'est traversé par aucun conflit, ce qui favorise peut-être la distanciation opérée par l'enseignant. La suite de la séance va confronter l'enseignant à un choix de posture: conserver une posture de lâcher-prise et laisser le groupe gérer seul le conflit social qui va le traverser, ou intervenir.

\section{Analyse des phénomènes intersubjectifs qui infiltrent les postures, à l'insu du professionnel}

C'est dans l'après-coup des situations, dans un cadre prévu à cet effet, que nous avons pu revenir avec l'enseignant sur l'analyse de la dimension transférentielle sous-tendant ses postures, en particulier à l'égard de Chloé. Ce travail nous a permis d'examiner plus largement le lien entre l'idéal et les affects ressentis.

Revenons à la séance précédemment évoquée.

Différentes questions émergent, sur lesquelles le groupe pourrait travailler: " comment fonctionne une éolienne?" (Laura), "quelle est son utilité? " (Joseph), " son coût?" (Julien), "où en trouve-t-on?" (Laura), "que signifie une énergie renouvelable?" (Lucie), "quel avenir pour les énergies renouvelables? " (Lucie). Chloé veille à distribuer la parole, à respecter le tour de chacun. Ce travail requiert un effort d'attention et une tension perceptible.

Après un bref temps de silence, pendant lequel les jeunes s'interrogent en euxmêmes, Amélie (experte) précise à Chloé (novice) qu'elle doit passer à l'étape suivante: la répartition des tâches. Chloé demande au groupe: " qui veut travailler 
sur le fonctionnement d'une éolienne? " Laura propose de travailler avec Joseph. Puis vient le tour de Lucie, de Laura et d'Amélie. Joseph note scrupuleusement les décisions. Alors qu'il reformule au groupe ce qu'il a noté, il s'arrête brusquement, gêné : " Julien, observateur; Laura et Joseph travaillent ensemble sur le fonctionnement, le coût et l'endroit où sont installées les éoliennes; Lucie, Marine et Amélie travaillent sur les énergies renouvelables en se rendant au CDI, et... Chloé? " Le groupe se met à rire: Chloé, très concentrée sur sa tâche (veiller au bon fonctionnement du groupe, à sa régulation), s'est en quelque sorte oubliée autant qu'elle l'a été par les autres. Mais Julien reprend: "Chloé, elle travaille ... seule!". C'est alors que l'enseignant intervient, du fond de la classe: " compte tenu des difficultés de Chloé pour se mouvoir et écrire, on ne peut pas envisager qu'elle soit seule. Ce n'est pas adapté. /l faut trouver une autre solution ". Lucie et Joseph proposent en chœur: "Laura peut travailler avec Chloé ". L'enseignant reprend: "Personne ne doit décider à la place d'un autre, il faut d'abord s'assurer que Laura est d'accord, c'est à elle et à elle seule de parler». Un silence suit ces paroles. Les regards se tournent vers Laura. C'est finalement Julien qui demande:

- Laura, es-tu d'accord?

- Non, je ne suis pas d'accord... Je ne sais pas comment dire cela, mais non.

Chloé reprend très vite, agitée: "on ne va pas t'en vouloir, si tu donnes ton avis". Brusquement et sèchement, l'enseignant s'adresse à Chloé: " on ne parle pas comme ça! Chacun se respecte ». Un silence de mort suit ces paroles. Finalement, c'est Amélie, visiblement mal à l'aise, qui rompt le silence: " si Chloé est d'accord, je travaille avec elle sur les énergies renouvelables".

L'analyse de cet épisode banal nous a permis, chercheur et praticien, de revenir ensemble sur nos interventions, en particulier sur notre propre absence de mots. Pourquoi s'adresser à Chloé comme si celle-ci avait commis une faute, alors même que l'adolescente reconnaissait à Laura la possibilité d'exprimer sa conflictualité et cherchait à la rassurer sur le maintien du lien (" tu peux donner ton avis, on ne t'en voudra pas ")?

C'est à partir de la reconnaissance des affects négatifs ressentis à l'égard de l'adolescente, et de leur transfert sur le groupe, qu'avec l'enseignant nous avons été amenés à réinterroger les idéaux et les valeurs, en particulier ceux d'un groupe imaginé comme rempart contre toute destructivité (y compris la sienne propre): "Le groupe doit à tout prix éviter aux jeunes d'être confrontés à l'échec, à la solitude, à la souffrance. Mon objectif premier: les amener à produire quelque chose avec d'autres, agir sur et avec d'autres, pour les sortir de leurs préoccupations douloureuses. Je pense que si les élèves découvrent qu'ils peuvent apporter à d'autres, ils verront qu'ils ne sont pas "si nuls", comme ils le disent souvent. Je voudrais ainsi les aider à sortir de leur passivité, à s'engager, à engager leur parole, à prendre des initiatives, à sortir d'eux-mêmes, à découvrir les bienfaits des liens de solidarité2. " La conviction qu'il suffit d'être dans le positif pour agir bien, a progressivement fait place à la prise en compte d'une réalité plus complexe, plus ambiguë. L'enseignant a été amené à effectuer une sorte de travail de deuil d'une réalité idéalisée, s'imposant

2. Cf. Entretien avec l'enseignant. 
de maintenir ensemble les contraires et traitant l'ambivalence humaine comme inéluctable.

Nous avons pu également identifier notre trouble à l'égard de cette jeune fille, trouble en grande partie lié à notre impuissance à la soulager. De même l'enseignant a reconnu, dans un second temps, sa propre difficulté à tolérer l'étrangeté de ce corps souffrant. Face à ce trouble et confronté à la culpabilité, l'enseignant a été amené à actualiser paradoxalement ses affects négatifs avec des justifications défensives, sans pouvoir en saisir toutes les implications sur soi, sur l'autre et sur le groupe. Nous avons également pris conscience qu'à notre insu, nous avions contribué, dans la séance décrite précédemment, à taire une dimension essentielle de l'expérience relationnelle qu'apporte le groupe: la possibilité de laisser s'exprimer la conflictualité dans le groupe, sans que le lien groupal s'en trouve détruit pour autant. L'expérience du collectif, revisitée dans sa dimension inconsciente avec la prise en compte de la dimension transférentielle, permet ainsi de mettre au jour les relations complexes entre le psychique et le social.

\section{CONCLUSION}

II s'agissait dans cet article d'analyser les enjeux d'un travail de mise en groupe dans un contexte d'enseignement en unité hospitalière pour des adolescents gravement malades ou blessés. Le contexte de groupe semi ouvert qu'induit la situation particulière des élèves malades, complexifie l'édification du sentiment interne de sécurité. Nous avons toutefois montré que la construction d'un objet groupe internalisé est malgré tout possible, grâce à l'étayage de l'enseignant, des pairs, à l'intériorisation par chacun des différents repères (espace, temps, personnes, attitudes), et à la satisfaction éprouvée lors des expériences collectives. Quels sont les principaux apports de cette recherche sur les apprentissages que les situations de groupe ont favorisés, en particulier du point de vue formatif?

Du point de vue formatif, le groupe pédagogique a promu à la fois une fonction intégrative, avec le développement de liens d'appartenance au groupe, une fonction d'individuation, avec l'acceptation et le développement de la diversité et de l'individualité dans le groupe, et enfin une fonction créative ou transformatrice, avec la découverte de nouvelles pensées, de nouvelles voies possibles.

Ainsi, pour chacun de ces jeunes malades, l'effort important réalisé pour entrer dans le monde du groupe s'est-il vu récompensé par l'expérience d'appartenance offerte comme soutien à la construction/reconstruction du sens de soi en tant qu'individu ayant inconditionnellement le droit de vivre et d'occuper un espace affectif. La reconnaissance de chaque membre du groupe comme occupant une position spécifique, en particulier grâce au travail d'observation institué par l'enseignant, a favorisé une acceptation et un développement de la diversité et de l'individualité dans le groupe. Cette évolution est comparable à un mouvement vers un sens plus développé et plus conscientisé de l'intersubjectivité. Parallèlement à ce progrès, nous avons pu constater un accroissement du degré d'initiative et d'activité chez la plupart des élèves, ainsi qu'une plus large tolérance à la frustration et aux contraintes internes et externes liées à la situation pédagogique. Comme si la reconnaissance des écarts entre soi et autrui, dans un contexte où le jeune réalise 
en même temps l'universalité des "manques en soi » et des nécessités de la réalité, favorisait l'acceptation de ses propres limites, manques ou doutes... L'individualité devient alors mieux assurée et moins intransigeante, l'élève se montre capable de transactions avec les autres, d'une plus grande tolérance à son égard, lorsqu'il doute, se trompe ou ne sait pas. La fonction d'individuation s'illustre également à travers le développement de l'estime de soi et du sentiment de compétence: ce que l'élève nomme aux autres, qui prend forme et sens grâce à son intervention dans l'espace du groupe pédagogique signe une création qui lui est propre, et lui est, en retour, reconnue socialement.

La fonction créative ou transformatrice s'est révélée lors des expériences plus délicates, voire douloureuses, que certains élèves, comme Chloé, ont vécues et que nous avons précédemment analysées. L'expérience de ne pas comprendre (décalage linguistique par exemple), de ne pas réussir à se faire comprendre ou entendre d'un environnement qui ne répond pas comme on s'y attend, force parfois le sujet, élève ou enseignant, à un dépassement de soi, pour recréer l'expérience de synchronie, d'accordage qui ne va pas de soi, ou bien parvenir à son objectif personnel.

Nous avons par ailleurs questionné les liens entre l'apprentissage des élèves, leurs éventuelles progressions au regard de l'expérience relationnelle qu'implique le groupe, et les postures de l'enseignant. Nous avons montré que les postures professionnelles relèvent, pour une part, de schèmes construits du " penser/dire/ faire ", visant intentionnellement l'étayage des apprenants. En nous référant à la perspective psychosociologique et développementale, nous observons que l'enseignant construit ses actes de parole et ses gestes professionnels dans un lien dynamique avec l'apprenant (intersubjectivité fonctionnelle entre enseignant/ apprenant) d'une part, en fonction des interactions entre élèves et de la dynamique du groupe d'autre part. L'exemple de Chloé nous a cependant ouvert à plus de complexité : la dimension éthique de nos actes professionnels est présente chaque fois que nos actes - en particulier nos actes de parole - rejaillissent sur ceux avec lesquels nous travaillons, enfants ou adultes, et que nos impasses, nos contradictions, notre ambivalence interrogent nos valeurs. Comme Didier Anzieu (1999) le précise, le groupe peut être érigé en objet libidinal, " avec le risque d'assigner aux participants comme but plus ou moins explicite d'avoir à le faire exister. Faire un groupe, faire un bon groupe ". Cet objectif constitue ici un déplacement défensif par rapport au but véritable, recherché mais redouté, de l'apprentissage: la transformation des acquis de chacun, qui passe inévitablement par un processus de mise en question personnelle et de confrontation aux limites et aux manques en soi. La tentation de faire que le groupe devienne la visée du groupe, pour restaurer collectivement des narcissismes individuels menacés, nous semble d'autant plus grande que nous devons faire face à des vécus traumatiques en lien avec la maladie. 


\section{Références}

Anzieu, D. (1999). Le groupe et l'inconscient. Paris: Dunod.

Bruner, J.S. (1991). Car la culture donne forme à l'esprit. Paris: EsHel.

Bucheton, D. et Soule, Y. (2009). Les gestes professionnels et le jeu des postures de l'enseignant dans la classe: un multi-agenda de préoccupations enchâssées. Éducation \& didactique, 3(3), 29-48.

Clot, Y. (2010). La fonction psychologique du travail. Paris: PUF.

Doise, W. et Mugny, G. (1981). Le développement social de l'intelligence. Paris: InterEditions.

Doudard, A., Riquin, É., Malka, J., Avarello, G. et Duverger, P. (2014). De la sidération à la remise en mouvement chez l'enfant malade. Comment faire avec le chaos? Enfances \& Psy, 64(3), 131-139.

Freud, S. (1981). Psychologie des foules et analyse du moi. Essais de psychanalyse. Paris: Payot, 83-175. (CEuvre originale publiée en 1921).

Gilly, M. (1995). Approches socio-constructives du développement cognitif de I'enfant d'âge scolaire. In D. Gaonach' et C. Golder (dir.), Manuel de Psychologie pour l'enseignement, (p. 130-167). Paris: Hachette.

Gutton, P. (1979). À propos du travail de la souffrance de l'enfant. Psychanalyse à l'université, 4(15), 435-444.

Kaës, R. (2005). La parole et le lien. Processus associatifs et travail psychique dans les groupes. Paris: Dunod.

Kaës, R. (2003). Réalité psychique et souffrances dans les institutions. In R. Kaës (dir.), L'institution et les institutions (p. 1-46). Paris: Dunod.

Kaës, R. (2002). Les théories psychanalytiques de groupe. Paris: PUF.

Korff-Sausse, S. (1996). Le miroir brisé. Paris: Calmann Lévy.

Oppenheim, D. (2003). Grandir avec un cancer. L'expérience vécue par l'enfant et l'adolescent. Paris: de boeck.

Pedinielli, J.-L. (1987). Le travail de la maladie. Psychologie médicale, 19(7), 1049-1052.

Pedinielli, J.-L. (1994). Hypothèse d'un travail de la maladie. Cliniques méditerranéennes, 41(42), 169-194.

Perret-Clermont, A.-M. et Nicolet, M. (2001). Interagir et connaître. Enjeux et régulations sociales dans le développement cognitif. Paris: L'Harmattan.

Pichon-Rivière, E. (1971). El proceso grupal. Del psicoanalisis a la psicologia social. Buenos Aires: Ediciones Nueva Vision s.a.i.c. Traduit par Le processus groupal, Toulouse, Érès (Ed. de 2004).

Rouchy, J.-C. (2009). De I'analyse de groupe. In F. Marty (dir.), Les grandes problématiques de la psychologie clinique (p. 195-220). Paris: Dunod.

Roux, J.-P. (2003). Le travail en groupe à l'école. Cahiers pédagogiques, 424.

Sirota, A. (2007). Analyser collectivement les situations éducatives. Une classe de seconde: de l'inquiétante étrangeté à la découverte de ses semblables. In F. Rey et A. Sirota (dir.), Des clés pour réussir au collège et au lycée (p. 235-251). Ramonville-Saint-Agne : Érès. 
Toubert-Duffort, D. (2009). L'éducation à l'autonomie des jeunes handicapés. In A. Weil-Barais (dir.), Violence à l'école: des violences vécues aux violences agies (p. 107-120). Paris: Bréal.

Toubert-Duffort, D. (2011). Écouter les corps en quête de sens: un travail groupal avec des adolescents atteints de lésions cérébrales. Revue de psychothérapie psychanalytique de groupe, 57, 105-119.

Toubert-Duffort, D. (2012). Apprendre en groupe, apprendre du groupe? Cliopsy, 7, 27-42.

Toubert-Duffort, D. et Sirota, A. (2016). Fraternel et CEdipien: enjeux identitaires. Adolescence, 34(3), 607-620.

Vygotski, L.S. (1997). Pensée et langage (réédition de la traduction française revue et modifiée de Françoise Sève). Paris: Éditions La Dispute. (CEuvre originale publiée en 1934).

Winnicott, D.-W. (1978). Jeu et réalité. Paris: Gallimard. (CEuvre originale publiée en 1971). 